\title{
Minichromosome maintenance protein in myxofibrosarcoma
}

\author{
James D Sington ${ }^{1}$, Alex Freeman ${ }^{1}$, Lesley S Morris ${ }^{1}$, Sarah L Vowler ${ }^{2}$, Barbara N Arch ${ }^{2}$, \\ Cyril Fisher ${ }^{3}$ and Nicholas Coleman ${ }^{1}$ \\ ${ }^{1}$ Department of Molecular Histopathology; ${ }^{2}$ Centre for Applied Medical Statistics, University of Cambridge, \\ Cambridge, UK and ${ }^{3}$ Department of Histopathology, Royal Marsden Hospital, London
}

\begin{abstract}
Histopathological assessment of myxofibrosarcoma may be difficult, especially on the basis of a small core biopsy, which enables only a crude evaluation of grade and prognosis. We have tested the hypothesis that determination of cell cycle state may assist in the diagnostic assessment of myxofibrosarcoma. We have studied 51 cases of high-grade $(n=20)$, intermediate-grade $(n=21)$, and low-grade $(n=10)$ myxofibrosarcomas, as well as nine cases of benign myxoma. Cell cycle state within tumors was determined by immunostaining for the recently described marker minichromosome maintenance protein 2 (MCM2), together with Ki67. Labelling indices for both markers were correlated with tumor grade, mitotic index, and time to first recurrence. The MCM2 labelling indices were significantly higher than the Ki-67 labelling indices. Both indices showed a significant correlation with the mitotic index and both showed significant increases with increasing grade of myxofibrosarcoma. The MCM2 labelling index (but not the Ki67 labelling index) showed a significant inverse exponential correlation with the time to first recurrence. Myxoid and cellular areas showed no difference in the MCM2 and Ki-67 labeling index, suggesting that clinically useful information could be obtained from any component of a myxofibrosarcoma sampled in a needle biopsy and/or cytological specimen. We therefore suggest that assessment of cell cycle state may be a useful diagnostic adjunct in the histopathological assessment of myxofibrosarcoma, by enabling more accurate determination of grade and prediction of outcome.
\end{abstract}

Modern Pathology (2004) 17, 235-240, advance online publication, 19 December 2003; doi:10.1038/modpathol.3800044

Keywords: myxofibrosarcoma; recurrence; prognosis; MCM2; Ki67

Myxofibrosarcoma is a common soft-tissue neoplasm, which most frequently occurs in the subcutaneous extremities of the elderly. Myxofibrosarcoma is regarded as a malignant fibroblastic lesion with nodular growth and variable myxoid stroma. Higher grades are equivalent to the myxoid variant of malignant fibrous histiocytoma. ${ }^{1}$ Mortality related to the tumor is low, although there is a very high rate of local recurrence, with at least $61 \%$ of cases being reported to recur locally. ${ }^{1-3}$ Mentzel et $a l^{3}$ have described a three-tier grading system, with high, intermediate, and low -grades of myxofibrosarcoma. However, grading of myxofibrosarcoma can be a difficult exercise, especially in a small needle biopsy where only the myxoid component may be present. ${ }^{4}$

Correspondence: Dr JD Sington, MB ChB, Department of Cellular Pathology, John Radcliffe Hospital, Oxford, OX3 9DU, UK.

E-mail: jamiesington@yahoo.co.uk

Received 25 March 2003; revised 12 August 2003; accepted 18

August 2003; published online 19 December 2003
Here, we have tested the hypotheses that evaluation of the cell cycle state of myxofibrosarcoma tumors may facilitate the assessment of grade in needle biopsies and/or cytological specimens and may also be predictive of outcome. In order to obtain an accurate indication of cell cycle state in myxofibrosarcoma, we have examined the expression of minichromosome maintenance (MCM) protein 2, which is a member of the prereplicative complex ${ }^{5-7}$ that serves as an essential licensing factor for DNA replication in eukaryotic cells. ${ }^{8}$ MCM proteins are detectable throughout the cell cycle but are lost in quiescence and differentiation. We have previously shown that expression of MCM proteins is a characteristic of dysplastic and malignant cells, and that antibodies against MCM proteins enable the identification of more cells in cycle than the existing proliferation markers Ki67 and proliferating cell nuclear antigen (PCNA). ${ }^{9,10}$ In all of our studies to date, antibodies against different MCM proteins have produced essentially identical results.

In the current study, we have quantified the expression of MCM2 and Ki67 in 51 cases of 
myxofibrosarcoma (20 high grade, 21 intermediate grade and 10 low grade) and nine cases of intramuscular myxoma. We have correlated MCM2 and Ki67 labelling indices with grade and mitotic index of myxofibrosarcoma and, for those tumors for which we could obtain follow-up data, with the time to local recurrence.

\section{Materials and methods}

\section{Clinical Specimens}

Paraffin blocks of 51 cases of myxofibrosarcoma and nine intramuscular myxomas were obtained from the files of the Royal Marsden Hospital. The cases of myxofibrosarcoma were divided into low, intermediate, and high-grade, according to the criteria of Mentzel et al. ${ }^{3}$ A separate mitotic count was performed on at least 10 high-power fields $\left(1 \mathrm{hpf}=0.16 \mathrm{~mm}^{2}\right)$ by a single observer. Follow-up data, including details of local recurrence, were available for 20 myxofibrosarcoma cases from the Royal Marsden Hospital database. No follow-up data were available for the other 31 myxofibrosarcoma cases, most of which were from patients treated in distant centers. The myxofibrosarcoma sections were stained by immunohistochemistry for the expression of MCM2 and Ki67, and the myxoma specimens were stained for the expression of MCM2.

\section{Immunohistochemistry}

Paraffin sections, $5 \mu \mathrm{m}$ thick, were cut onto aminopropyltriethoxysilane-coated slides, dewaxed in xylene, and taken through a series of ethanols to water. The tissues were pressure-cooked in $0.08 \mathrm{M}$ citrate buffer for 2 or $10 \mathrm{~min}$ for antigen retrieval. Following washes in Tris-buffered saline (TBS), endogenous peroxidase activity was quenched by incubation in $0.6 \%$ hydrogen peroxidase in TBS for $30 \mathrm{~min}$. Sections were then washed in TBS and blocked with $10 \%$ goat serum (DAKO, Ely, UK) in TBS for $2 \mathrm{~h}$.

Antibodies were diluted in TBS containing 1\% BSA and $0.1 \%$ Triton $\mathrm{x}-100$. A volume of $100 \mu \mathrm{l}$ of antibody was added to each section, and the slides were incubated at $4^{\circ} \mathrm{C}$ overnight in a humidified chamber. Optimal staining was found with antiMCM-2 hybridoma supernatant at 1:15 and with anti-Ki67 (MIB-1, Immunotech) at 1:100.

The slides were then washed in TBS containing $0.025 \%$ Triton X-100 and incubated for $1 \mathrm{~h}$ with biotinylated goat anti-rabbit secondary antibody (DAKO). A streptavidin-horseradish peroxidase system (DAKO) with the substrate diaminobenzidine was used to develop the stain. The stains were lightly counterstained with Harris' hematoxylin, dehydrated in ethanol, and cleared in xylene.
Coverslips were applied with DEPEX mounting medium (Gurr, BDH, Poole, Dorset, UK).

\section{Quantification of Immunohistochemical Staining}

A quantitative indication of the extent of staining was obtained for each case by calculating the proportion of cells expressing MCM2 and Ki67 in randomly selected microscopic fields. Between 500 and 1000 nuclei were assessed per case by a single observer. Results were expressed as a labelling index, being the percentage of nuclei in representative microscopic fields that were positively stained. In six randomly selected cases of myxofibrosarcoma (three high-grade and three intermediate-grade), a comparison was made between the labelling indices in the myxoid and cellular areas of stained sections.

\section{Statistical Analysis}

Differences between MCM2 and Ki67 labelling indices, and between labelling indices in myxoid and cellular areas of individual sections were assessed using the nonparametric Wilcoxon signed rank test. Differences in labelling indices between grades of myxofibrosarcoma were assessed using the Mann-Whitney U-test. In all cases, the $P$ values were two-tailed. The correlations between labelling indices, mitotic index, and time to recurrence for each grade of myxofibrosarcoma were evaluated using Spearman's correlation coefficient.

\section{Results}

The 51 cases of myxofibrosarcoma comprised 20 high-grade (39\%), 21 intermediate-grade (41\%), and 10 low-grade $(20 \%)$ tumors. The mean patient age was 66 years (range 40-93 years) and the female: male ratio was 1.1:1. Data on site of occurrence were available for 44 cases: 33 (75\%) of these occurred in proximal or distal limbs, with the remainder occurring in trunk, head and neck, and retroperitoneum.

All cases of myxofibrosarcoma showed strong nuclear expression of MCM2 (Figure 1) and Ki67, with no variation in labelling indices in different regions of individual tumors. In particular, the myxoid and cellular areas of the myxofibrosarcoma tumors showed no significant difference in the MCM2 labelling index or Ki67 labelling index $(P=0.125$ for both). In all myxofibrosarcoma tumors, the MCM2 labelling index was higher than the Ki67 labelling index (Table $1, P<0.001$ ). The mitotic index showed a strong correlation with the MCM2 labelling index for all myxofibrosarcoma grades and for the myxofibrosarcoma tumors as a group (Figure 2, $r=0.763, P<0.001$ ). There was also a correlation between the mitotic index and the Ki67 

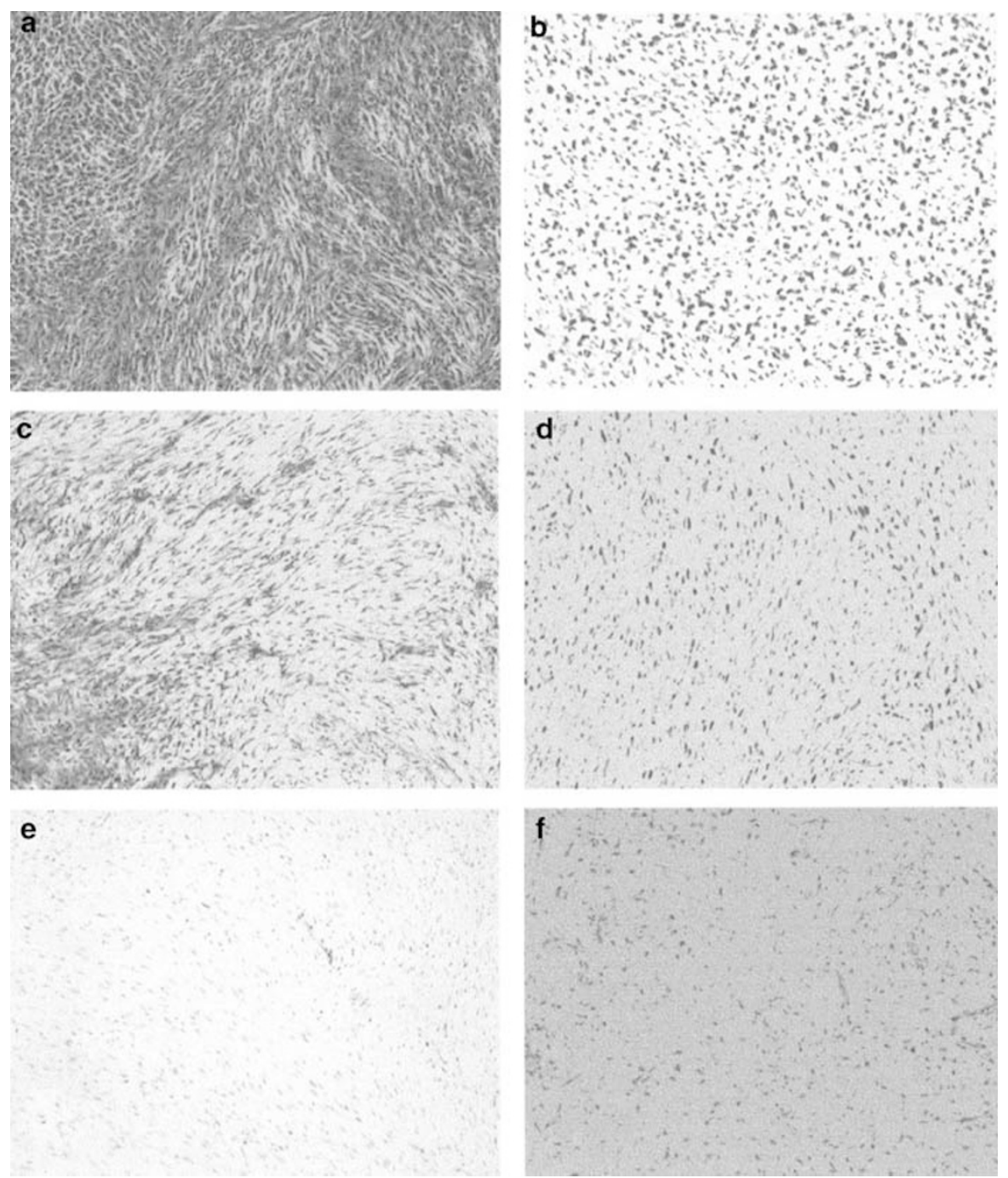

Figure 1 Histology and immunohistochemical demonstration of MCM2 in myxofibrosarcoma; (a,b) high-grade myxofibrosarcoma, (c,d) intermediate-grade myxofibrosarcoma, (e,f) low-grade myxofibrosarcoma.

Table 1 Medians and interquartile (IQ) ranges for MCM2 labelling index (LI) Ki67 labelling index (LI), mitotic rate (1 hpf $=0.16 \mathrm{~mm}{ }^{2}$ ) and evidence of local recurrence

\begin{tabular}{|c|c|c|c|c|}
\hline & High grade & Intermediate grade & Low Grade & Мухота \\
\hline Total number & 20 & 21 & 10 & 9 \\
\hline MCM LI & 0.75 & 0.57 & 0.27 & 0.04 \\
\hline (median and IQ range) & $0.64,0.89$ & $0.46,0.67$ & $0.13,0.33$ & $0.03,0.05$ \\
\hline Ki67 LI & 0.51 & 0.32 & 0.10 & - \\
\hline (median and IQ range) & $0.42,0.58$ & $0.20,0.38$ & $0.05,0.14$ & - \\
\hline Mitotic rate $/ 10 \mathrm{hpf}$ & 23.5 & 8 & 1 & - \\
\hline (median and IQ range) & 16,46 & 5,13 & 0,1 & - \\
\hline Number with available follow-up data & 5 & 12 & 3 & - \\
\hline Time to first recurrence (months) & 11 & 24 & 60 & - \\
\hline (median and range) & $2-36$ & $4-72$ & $24-96$ & - \\
\hline
\end{tabular}

labelling index for the myxofibrosarcoma tumors as a group $(r=0.815, P<0.001)$.

The MCM2 and Ki67 labelling indices increased with increasing grade of myxofibrosarcoma (Figure 2).
There were significantly higher labelling indices for MCM2 and Ki67 in high-grade compared to intermediate-grade myxofibrosarcomas and in intermediate-grade compared to low-grade myxofibrosarcomas 


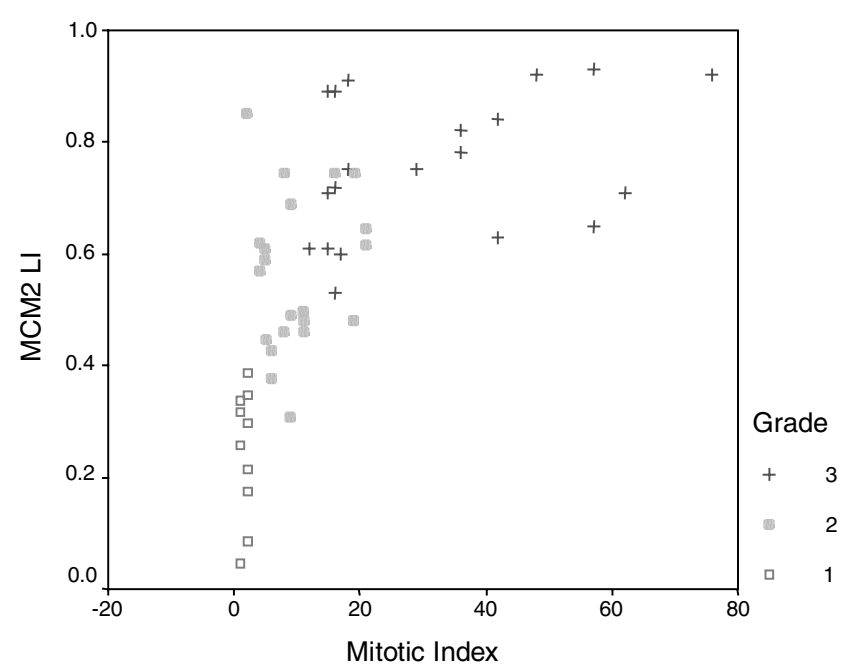

Figure 2 Comparison of mitotic index and MCM2 labelling index (LI) in myxofibrosarcoma (cc $=0.763, P<0.001,1=$ low grade, $2=$ intermediate grade, $3=$ high grade). Mitotic index represents the number of mitoses per 10 high-power fields $(1 \mathrm{hpf}=$ $0.16 \mathrm{~mm}^{2}$ ).
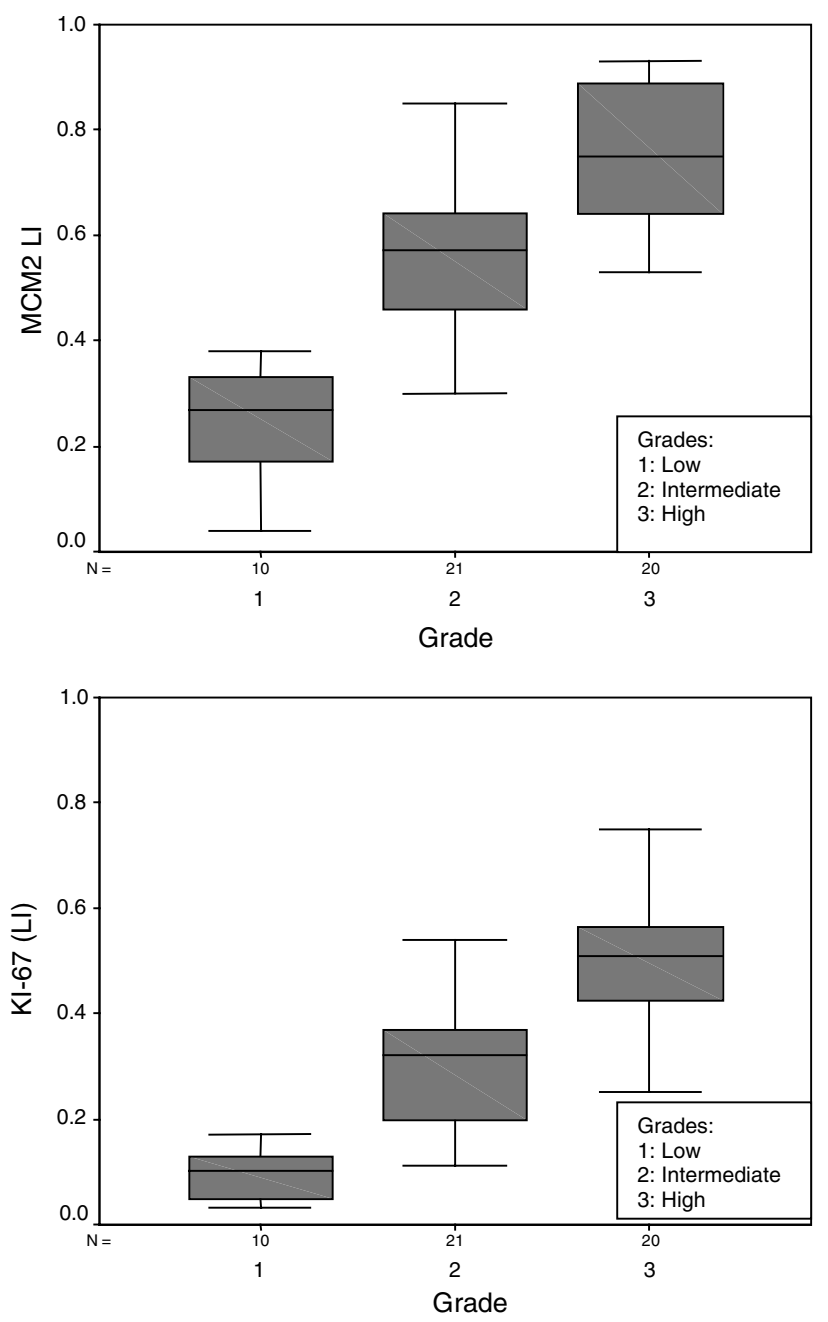

Figure 3 MCM2 and Ki67 labelling indices for each grade of myxofibrosarcoma. Horizontal line = median; box =interquartile range.

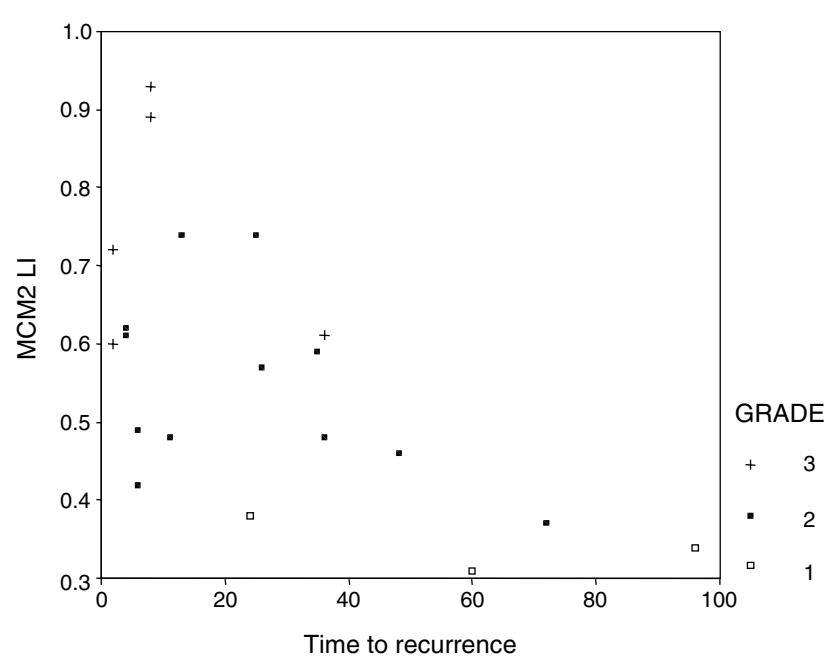

Figure 4 Comparison of MCM2 labelling index and time to recurrence in different grades of myxofibrosarcoma ( $\mathrm{cc}=-0.559$, $P=0.01,1=$ low grade, $2=$ intermediate grade, $3=$ high grade).

( $P<0.001$ in all cases). There was no overlap between the MCM2 or Ki67 labelling indices for high-grade and low-grade myxofibrosarcoma (Figure 3). The MCM2 labelling index was significantly higher in low-grade myxofibrosarcoma compared to myxoma $(P<0.001)$.

We were able to obtain follow-up data for 20 cases of myxofibrosarcoma. In all of these cases, local recurrence had occurred, over a period that ranged from 2 to 96 months. There was no significant correlation between the time to recurrence and the Ki67 labelling index $(r=-0.29, P=0.214)$ or the mitotic index $(r=-0.378, P=0.10)$. In contrast, there was an inverse exponential correlation between the time to recurrence and the MCM2 labelling index (Figure 4) $(r=-0.559, P=0.01)$. Indeed, all tumors showing MCM2 labelling index values above $0.50(n=11)$ recurred in less than 3 years.

\section{Discussion}

MCM2 is a component of the prereplicative complex, ${ }^{7}$ that is an essential licensing factor for DNA replication and which limits replication to once per cell cycle. ${ }^{8,11}$ MCM proteins are highly sensitive and specific markers of cells that are in cycle (regardless of the rate of cycling) and therefore indicate the cell cycle state within a tissue. In all of our studies to date, staining for MCM proteins has identified a higher percentage of cells in normal, dysplastic, and neoplastic tissues than have the traditional proliferation markers Ki67 and PCNA. ${ }^{9,10}$

The results of our present study suggest that determination of cell cycle state may help in assessment of grade in myxofibrosarcomas, particularly in needle biopsies or cytological specimens in 
which only small numbers of neoplastic cells may be present. Both the MCM2 labelling index and the Ki67 labelling index appear to provide useful information in myxofibrosarcoma, although the MCM2 labelling index is of particular value.

Our series of myxofibrosarcomas contained a higher percentage of intermediate-grade tumors (41 vs $24 \%$ ) and a lower percentage of high-grade tumors (39 vs $56 \%$ ) than previously reported series, ${ }^{3}$ although there was no difference in the frequency of low-grade tumors $(20 \%)$. In all grades of myxofibrosarcoma examined, the MCM2 labelling index was significantly higher than the Ki67 labelling index. Both the MCM2 labelling index and the Ki67 labelling index were significantly higher in increasing grades of myxofibrosarcoma, the Ki67 data confirming the previous findings of Mentzel et al. ${ }^{3}$ We observed no overlap of the MCM2 or Ki67 labelling index values between high-grade and lowgrade myxofibrosarcomas. Indeed, our results suggest that determination of the MCM2 labelling index for an individual case would enable the assignment of that case into a high- or intermediate-grade category (MCM2 labelling index greater than 0.5) or into a low- or intermediate-grade category (MCM2 labelling index less than 0.5). An investigation of the reproducibility of this finding in an independent series of specimens is warranted.

Our data also suggest that determination of the MCM2 labelling index would be of value in the assessment of needle biopsies and/or cytological samples from myxofibrosarcoma tumors. We observed that the MCM2 labelling index and Ki67 labelling index were uniform across sections of individual myxofibrosarcoma lesions. In particular, the myxoid and cellular areas of high- and intermediate-grade myxofibrosarcoma showed no significant difference in MCM2 or Ki67 labelling indices. Determination of these labelling indices may therefore be of use in the grading of small needle biopsies and cytological samples from myxofibrosarcoma tumors, in which either myxoid or cellular regions may be sampled and where a relatively low number of nuclei may be available for staining. ${ }^{4}$

Analysis of cell cycle state may be of less value in assisting in the differential diagnosis of low-grade MFS from benign myxoma. This can be a difficult exercise morphologically, especially for those myxomas showing increased cellularity and vascularity. ${ }^{2}$ Although significantly higher MCM2 labelling indices were seen by us in low-grade myxofibrosarcomas compared to benign myxomas, there was overlap of the labelling index values for the two specimen groups. All cases with MCM2 labelling index of less than 0.04 in our study were myxomas rather than low-grade myxofibrosarcoma, although these tumors represented only three of the nine myxomas studied.

We have also demonstrated in this study that determination of MCM2 labelling index may facilitate prediction of outcome in myxofibrosarcoma.
Our observations that the Ki67 labelling index and mitotic index are not predictive of outcome are supportive of findings from earlier studies. In a previous investigation of lesions designated malignant fibrous histiocytoma, the Ki67 labelling index was observed to correlate with histological grade but not with prognosis ${ }^{12}$ and Mentzel et $a l^{3}$ found no association between the clinical course of myxofibrosarcoma and tumor grade or Ki-67 labelling index. ${ }^{3}$ In contrast to these findings, we observed a significant inverse correlation between the MCM2 labelling index and the time to first recurrence of myxofibrosarcoma. In our study, all myxofibrosarcoma tumors with an MCM2 labelling index above 0.5 ( $n s=11)$ recurred within 3 years of the initial diagnosis. We believe that a prospective analysis of the utility of MCM2 immunostaining in predicting outcome in myxofibrosarcoma is indicated.

In conclusion, we have shown that assessment of cell cycle state using antibodies against MCM2 or Ki67 may be of value in the histopathological examination of myxofibrosarcoma. Both markers may assist with the prediction of grade in needle biopsies or cytological specimens from myxofibrosarcoma tumors, regardless of which lesional component is sampled. In addition, immunostaining for MCM2 may facilitate the prediction of the clinical course of individual tumors. Further testing of the hypothesis that analysis of cell cycle state may be of value in the diagnostic assessment of myxofibrosarcoma is now warranted.

\section{Acknowledgment}

This work was supported by a grant from Cancer Research UK.

\section{References}

1 Weiss SW, Enzinger FM. Myxoid variant of malignant fibrous histiocytoma. Cancer 1977;39:1672-1685.

2 Graadt van Roggen JF, Hogendoorn PCW, Fletcher CDM. Myxoid tumours of soft tissue. Histopathology 1999;35:291-312.

3 Mentzel T, Calonje E, Wadden C, et al. Myxofibrosarcoma: clinicopathological analysis of 75 cases with emphasis on the low-grade variant. Am J Surg Pathol 1996;20:391-405.

4 Kilpatrick SE, Ward WG, Bos GD. The value of fineneedle aspiration biopsy in the differential diagnosis of adult myxoid sarcoma. Cancer (Cancer Cytopathol) 2000;90:167-177.

5 Kersey SE, Labib K. MCM proteins: evolution, properties and role in DNA replication. Biochim Biophys Acta 1998;1398:113-136.

6 Labib K, Tercero JA, Diffley JF. Uninterrupted Mcm2-7 function required for DNA replication fork progression. Science 2000;288:1643-1647.

7 Ritzi M, Baack M, Musahl C, et al. Human minichromosome maintenance proteins and human origin 
recognition complex 2 protein on chromatin. J Biol Chem 1998;273:24543-24549.

8 Takisawa H, Mimura S, Kubota Y. Eukaryotic DNA replication: from pre-replication complex to initiation complex. Curr Opin Cell Biol 2000;12:690-696.

9 Freeman A, Morris LS, Mills AD, et al. Minichromosome maintenance proteins as biological markers of dysplasia and malignancy. Clin Cancer Res 1999;5:2121-2132.
10 Williams GH, Romanowski $\mathrm{P}$, Morris LS, et al. Improved cervical smear assessment using antibodies against proteins that regulate DNA replication. Proc Natl Acad Sci USA 1998;95:14932-14937.

11 Tye BK. MCM proteins in DNA replication. Annu Rev Biochem 1999;68:649-686.

12 Zehr RJ, Bauer TW, Marks KE, Weltevreden A. Ki67 and grading of malignant fibrous histiocytomas. Cancer 1990;66:1984-1990. 